\title{
Caracterización psicosocial en una muestra de mujeres víctimas de violencia de género en Colombia: un estudio piloto
}

Psychosocial characterization in a sample of women victims of gender-based violence in Colombia:

a pilot study

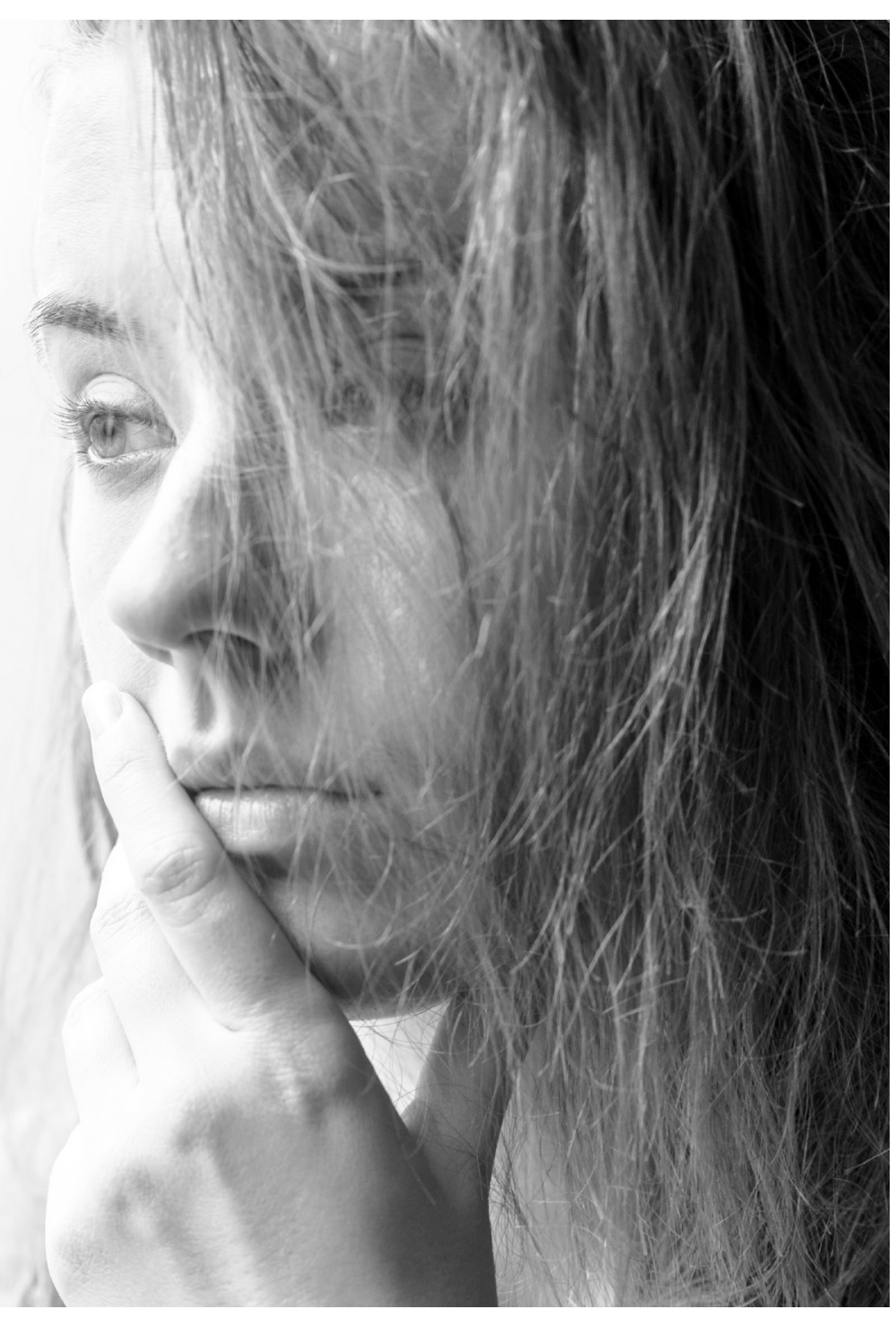




\title{
Caracterización psicosocial en una muestra de mujeres víctimas de violencia de género en Colombia: un estudio piloto ${ }^{1}$ \\ Psychosocial characterization in a sample of women victims of gender-based violence in Colombia: a pilot study
}

\author{
Edna Johanna Herrera Merchán², Dora Cristina Cañas Betancourt², Emily Carolina González \\ Clemente $^{4}$, Cirly Uribe Ochoa ${ }^{5}$, Lauren Audrey Padilla Reyes ${ }^{6}$
}

Artículo recibido en diciembre 5 de 2019; artículo aceptado en junio 8 de 2020

Este artículo puede compartirse bajo la Licencia Creative Commons Atribución-NoComercial-Compartirlgual 4.0 Internacional y se referencia usando el siguiente formato: Herrera, E. J., Cañas, D. C., González, E. C., Uribe, C. y Padilla, L. A. (2020). Caracterización psicosocial en una muestra de mujeres víctimas de violencia de género en Colombia: un estudio piloto. I+D Revista de Investigaciones, 15 (2), 135-147. DOI: https://doi.org/10.33304/revinv.v15n2-2020012

\section{Resumen}

En esta investigación se estudia el perfil psicosocial de una muestra de mujeres víctimas de violencia de género convocadas por la Fundación Mujer y Futuro, con edades entre los 19 y los 70 años. Para ello, se utilizó como instrumento una encuesta psicosocial, en la que se recolectó información sobre factores socioculturales y tipologías de violencia de género. El análisis de sistematización de datos de tipo descriptivo se hizo con el software estadístico SPSS (versión 24). Como resultado se encontró que un $70 \%$ de las mujeres de la muestra cuenta con estudios primarios y/o secundarios, el $65 \%$ es ama de casa, el $70 \%$ no cuenta con remuneración económica y el 90 \% se ubica en los estratos socioeconómicos uno y dos. En cuanto al tipo de violencia que han recibido, el $80 \%$ de las mujeres encuestadas ha sufrido violencia desde el hogar núcleo; el $100 \%$, psicológica; el $80 \%$, física; el $70 \%$, económica, y un $45 \%$, sexual. Además, a partir del contraste de los resultados con otras investigaciones, se concluye que la baja escolaridad, nula remuneración económica, el estrato socioeconómico y la violencia en el núcleo del hogar son

\footnotetext{
'Artículo científico empírico no experimental, de enfoque cualitativo, resultado de un proyecto de investigación terminado, perteneciente al área de ciencias sociales, subáreas de psicología social, derecho y género, y legislación de igualdad de género. Desarrollado en el grupo de investigación "Familia y Sociedad", Facultad de Psicología y grupo de investigación Los Mediadores, Facultad de Derecho, fue financiado por la Universidad Cooperativa de Colombia (Bucaramanga, Colombia). Dirección: carrera 31 con calle 32. PBX: 6854500. Fecha de inicio: agosto de 2016. Fecha de terminación: julio de 2018.

${ }^{2}$ Ph. D. en Neuropsicología Clínica, Facultad de Investigaciones Universidad Manuela Beltrán. Vinculada al Grupo de Investigación en Ciencias Sociales, Humanidades y Educación (CISNHE), Universidad Manuela Beltrán (Bucaramanga, Colombia). Dirección: calle 33 n. 27-12. PBX: 6525202. ORCID ID: https://orcid.org/0000-0002-1002-4044. Correo electrónico institucional: edna.herrera@docentes.umb.edu.co.

${ }^{3}$ Magíster en Psicología con énfasis en Cognición y Desarrollo Moral, Facultad de Psicología, Universidad Cooperativa de Colombia. Vinculada al Grupo de Investigaciones Salud y Sociedad. Universidad Cooperativa de Colombia (Bucaramanga, Colombia). Dirección: carrera 31 con calle 32. PBX: 6854500. ORCID ID: https://orcid.org/0000-0003-2954-1241. Correo electrónico institucional: dora.cañas@campusucc.edu.co.

${ }^{4}$ Magíster en Psicología Clínica y de la Salud, Facultad de Psicología, docente medio tiempo de la Universidad de Investigación y Desarrollo (Bucaramanga, Colombia). Dirección: calle 9 n. 23-55 PBX: 6352525. ORCID ID https://orcid.org/0000-0001-7472-0789. Correo electrónico institucional: egonzalez5@udi.edu.co.

${ }^{5}$ Magíster en Historia, Facultad de Derecho, Universidad Cooperativa de Colombia. Vinculado al Grupo de Investigación Los Mediadores. Universidad Cooperativa de Colombia (Bucaramanga, Colombia). Dirección: carrera 31 con calle 32. PBX: 6854500. ORCID ID: https://orcid.org/0000-0002-58943795. Correo electrónico institucional: cirly.uribeo@campusucc.edu.co.

${ }^{6}$ Magíster en Educación. Facultad de Psicología. Docente tiempo completo Universidad de Investigación y Desarrollo (Bucaramanga, Colombia). Dirección: calle 9 n.o 23-55. PBX:6352525. ORCID ID: https://orcid.org/0000_0001_7815_0672. Correo electrónico institucional:Ipadilla1@udi.edu.co.
} 
posibles factores vulneradores para el desarrollo de violencias.

Palabras clave: Caracterización psicosocial, mujeres, salud mental, salud pública, violencia de género.

\begin{abstract}
This research study analyzes the psychosocial profile of a sample of women victims of gender violence called by the Fundación Mujer y Futuro, aged between 19 and 70 years. To this end, a psychosocial survey in which information was collected on sociocultural factors and typologies of gender violence was used. The descriptive data systematization analysis was done with the statistical software SPSS (version 24). As a result, it was found that $70 \%$ of the women in the sample have primary and/or secondary studies, $65 \%$ are housewives, $70 \%$ do not have economic remuneration, and $90 \%$ are in socioeconomic strata one and two. In terms of the type of violence they have received, $80 \%$ of the women surveyed have suffered violence from the core of the home, $100 \%$, psychological, $80 \%$, physical, $70 \%$, economic, and $45 \%$, sexual. In addition, based on the contrast of the results with other investigations, it is concluded that low schooling, no economic remuneration, socioeconomic status, and violence in the nucleus of the home are possible vulnerability factors for the development of violence.
\end{abstract}

Keywords: Psychosocial characterization, women, mental health, public health, gender violence.

\section{Introducción}

En la literatura se reconoce que la violencia de género daña la salud mental y física de muchas mujeres en el mundo, pues son las únicas víctimas de dicho tipo de violencia (Bell et al., 2012; Vieyra et al., 2009). La magnitud del daño social ha generado un problema de salud pública importante, y por ello es necesario ahondar en la investigación de este tipo de tópicos, para obtener datos que permitan ayudar a mitigar este flagelo (Sanz y Sellarés, 2010). Por tales motivos, este artículo es un esfuerzo grupal de investigadores por mostrar una caracterización de mujeres víctimas de violencia de las que más adelante se desglosará un perfil psicosocial. Se cuenta con pocas publicaciones sobre esta temática en el estado colombiano y más en Bucaramanga, puesto que la mayoría de estudios de género se ha desarrollado más desde profesiones interdisciplinarias del derecho, la economía y la antropología (Rodriguez y lbarra, 2013). En la psicología aún falta profundizar también sobre las intervenciones en mujeres víctimas de la violencia (Guaderas Alburja, 2015; Sánchez et al., 2013); por tanto, se requiere mayor publicación por parte de la psicología en estos tópicos.

En Colombia esta realidad es endémica: las mujeres son violentadas de múltiples formas, tanto en el ámbito público como en el privado. Según datos del Instituto de Medicina Legal y Forenses, cada día son violentadas 100 mujeres por sus parejas o exparejas (Lozano, 2016), y esto es tan solo un tipo de violencia de género en el país.

Esta problemática, que afecta física y psicológicamente a la sociedad, se considera de gran relevancia, y es por ello que este estudio pretende realizar una caracterización psicosocial de mujeres víctimas de violencia de género en Colombia, a fin de conocer el nivel educativo y económico de estas, así como los tipos de violencia que han sufrido, la edad de inicio de recepción de violencia y la problemática familiar, además de contrastar las variables psicosociales estudiadas con otras investigaciones, lo que podría dar respuesta a situaciones de vulnerabilidad en estas mujeres.

El artículo expone a modo introductorio el marco teórico de la violencia, destacando los daños que ocasiona en las victimas, seguido de los conceptos de las diferentes violencias de género. Posteriormente se analizan los resultados obtenidos sobre la violencia de género mediante la aplicación de una encuesta psicosocial, lo que evidencia la tipología de malos tratos que ha vivido la muestra de mujeres evaluada, el tipo de agresor y la violencia en la familia núcleo. Finalmente, se presenta la discusión de los resultados con otros estudios, y se concluye que la violencia psicológica es la más frecuente, seguida de la física y la económica, y los bajos niveles educativos podrían ser un factor de vulnerabilidad de las violencias de género, de acuerdo con el análisis de la literatura. Es relevante la necesidad que existe de estudiar, indagar y actuar en este tipo de fenómenos que generan un daño irreversible a la sociedad.

\section{Marco teórico}

La violencia de género es una problemática presente en el contexto colombiano, que afecta a diferentes mujeres en distintos ámbitos sociales. Esta se caracteriza porque el cónyuge o pareja ocasiona daño en el área psicológica, física, sexual, social, económica o laboral de la mujer, lo que dificulta su bienestar integral; por 
tanto, es definida como "cualquier acto o intención que origina daño o sufrimiento físico, sexual o psicológico a las mujeres, incluyendo las amenazas de dichos actos, la coerción o privación arbitraria de libertad, ya sea en la vida pública o privada" (González y Bejarano, 2014, p. 425). Este tipo de violencia referida específicamente hacia la mujer implica una violación de los derechos humanos, ya que directamente repercute en la dignidad, integridad física, moral, seguridad, autonomía y libertad de la mujer, lo que la ubica como un fenómeno social de origen patriarcal, que implica un mecanismo de discriminación y subordinación. La violencia de género perpetúa la desigualdad infringida desde la negación de los derechos humanos, mediante la existencia de relaciones injustas de poder (Añón, 2016).

Para describir el control y la violencia que se ejerce hacia la víctima, Navarro (2015) expone el concepto de violencia coercitiva, que es el intento de ejercer control en lo que piensa, siente y hace la pareja, acompañado del deseo de hacerle daño, lo que incluye las agresiones físicas, amenazas, intimidación, culpabilización, utilización de los hijos y maltrato psicológico. Este tipo de violencia suele ser la que pone en mayor riesgo a la mujer, y se asocia al feminicidio, culmen del continuum de violencia. De hecho, el riesgo de feminicidio podría incrementarse ante situaciones de extrema vulnerabilidad social, como ser dependiente (económica y/o afectivamente) del agresor, pertenecer a una minoría étnica, padecer alguna enfermedad o tener un hijo de una unión previa (Contreras, 2014). Estas relaciones influidas por violencias coercitivas o situacionales terminan generando un grave problema de salud pública (Sanz y Sellarés, 2010).

No obstante, las cifras que demuestran el daño social ante casos de feminicidio y violencia de género conyugal son alarmantes. Según el Instituto de Medicina Legal y Ciencias Forenses, se perdieron 339.552 años de vida saludable, $52,7 \%$ (178.883) en mujeres y 47,3 $\%$ (160.669) en hombres, que pierden más años de vida saludable por lesiones en violencia interpersonal, en tanto que las mujeres lo hacen en episodios de violencia de pareja (Lozano, 2016, p. 15). En cuanto a las circunstancias, las cifras del Instituto de Medicina Legal y Forense indican que "en el caso de las mujeres tenemos la violencia intrafamiliar con un $28,47 \%$, el feminicidio con un $19,06 \%$ y la violencia interpersonal con un 15,84 \%" (Lozano, 2016, p. 77), con lo cual se considera que "el principal riesgo para la vida de las mujeres en Colombia no es el conflicto armado, sino las relaciones personales establecidas desde una cultura patriarcal" (Lozano, 2016):

El escenario de los hechos nos dice que la vivienda es el lugar donde más asesinan a las mujeres, en un $33,27 \%$, con 335 casos; en segundo lugar, está la vía pública, en un $32,77 \%$, con 330 casos; en tercer lugar, los espacios terrestres al aire libre, con un 7,25\%, con 73 casos. (Lozano, 2016, p. 79)

El que la vivienda sea el lugar más peligroso para las mujeres lo ratifica también el rango de horario en que las mujeres son violentadas, puesto que, según el mismo organismo, el "horario comprendido entre las 21:00 y las 23:59 es aquel en el que más casos de asesinatos en mujeres se dieron en el año 2018. De las 9:00 a las 11:59 fue cuando menos casos se registraron" (p. 81), tal vez porque en este último horario ellos no están en casa. "En el año 2018 se realizaron 49.669 peritaciones en el contexto de la violencia de pareja, cuya tasa es de 120,57 casos por cada cien mil habitantes, siendo el hombre el principal presunto agresor" (p.199).

También el Observatorio de Igualdad de Género de America Latina y el Caribe (2018) expone que en el año 2017 se evidenció un aumento de 2795 casos de feminicidios en América Latina y el Caribe con relación a 2012; crímenes efectuados en su mayoría por familiares, parejas o exparejas de la mujer víctima. Estas cifras muestran el aumento en la vulneración de los derechos de las mujeres a una vida libre de violencia, y es importante reconocer los daños en salud mental y cognitivos que se derivan de dicha problemática.

Un acto violento podría generar síntomas ansiosos e incluso ser causante de un trastorno de estrés postraumático que ocasione en la víctima una reviviscencia continua de los episodios de violencia, incluso encontrándose fuera de peligro. Adicionalmente, la ansiedad puede presentarse en comorbilidad con episodios depresivos y psicóticos (Andrade et al., 2013; Cea, 2015; Vieyra et al., 2009), trastornos disociativos, riesgos suicidas, consumo de sustancias y alcoholismo, e incluso ser precipitante de algunos trastornos de ansiedad, como los obsesivo-compulsivos y/o ansiedad generalizada (Navarro, 2015; Ribeiro et al., 2009). Esto indica que las repercusiones en la salud mental son elevadas e interfieren de forma costosa en la vida normal de la víctima (Bell et al., 2012; Hewitt et al., 2016; Vieyra et al., 2009).

\section{Tipología de las violencias}

En cuanto a los tipos de violencia que se han conceptualizado taxonómicamente, se encuentran la violencia psicológica, física, económica, social, patrimonial y/o vicaria, laboral, institucional y por redes sociales, todas estas comprendidas en marcos legales y protocolos de atención que han permitido entender los orígenes de dichas violencias (Walker, 2012).

Al ahondar en la definición de los tipos de violencia mencionados, se encuentra que la violencia psicológica es concebida como la pretensión de causar daño o 
sufrimiento emocional o psicológico. Dentro de esta categoría se encuentran las humillaciones, insultos, amenazas de muerte, malos tratos verbales, bromas hirientes, vigilancia constante, negación de la dignidad e inseguridad, con el fin de generar una dependencia emocional y pérdida de la autoestima (Chejter e Isla, 2018; Ruta pacífica de las mujeres, 2013; Servicio Andaluz de Salud, 2019; Walker, 2012).

Otro tipo de violencia es la física, conceptualizada como cualquier forma de contacto físico para someter, controlar o intimidar. Dentro de esta categoría están los puñetazos, empujones, bofetadas, golpes con armas u otros objetos, patadas, etc. (Walker, 2012).

Por otra parte, se encuentra la violencia sexual, definida como las conductas que coaccionan o fuerzan a la mujer (en este caso) a realizar o soportar actos de tipo sexual en contra de su voluntad. En esta categoría se encuentran el acoso sexual (comportamientos intimidatorios como tocamientos sexuales a cambio de favores económicos, ayudas o ascensos laborales, o mejoras académicas), el acceso carnal violento, configurado como una agresión altamente profanativa de tipo sexual que termina con un coito en contra de la voluntad de la mujer, así exista la condición de matrimonio (Walker, 2012), y la explotación sexual, conceptualizada como la captura o atracción hacia la víctima con la intención de comercializarla y explotarla sexualmente. Dentro de los hogares también es concebida como la utilización de la figura femenina (niña) por parte de un progenitor, padrastro o proveedor económico de la familia para su abuso (Orjuela y Rodríguez, 2012).

Otra categoría de violencia sexual es el embarazo forzado, producto de un acceso carnal violento que promueve las situaciones de daño emocional, psicológico y físico en la víctima (Chejter e Isla, 2018). También se encuentra como categoría de violencia sexual la negación de la anticoncepción, puesto que no permite un control de natalidad en las víctimas para planear su vida familiar (Chejter e Isla, 2018), y, por último, el incesto, que como todo tipo de violencia sexual es la apropiación violenta y directa del cuerpo de la mujer y su sexualidad, con el agravante de la consanguinidad. En escenarios donde opera el conflicto armado, es una violencia sociosexual del sistema patriarcal que se agudiza e incrementa lo que genera la humillación, destrucción de la seguridad y estima propia de las mujeres (Ruta pacífica de las mujeres, 2013).

Por su parte, la violencia económica se refiere a:

[...] los comportamientos que incluyen la privación intencionada y no justificada legalmente de recursos para el bienestar de la mujer y de sus hijos e hijas, o la discriminación en la disposición de los recursos compartidos en el ámbito familiar, en la convivencia de pareja o en las relaciones posteriores a la ruptura de la misma. (Servicio Andaluz de Salud, 2019, p. 446)

En cuanto a la violencia social hacia la mujer, esta es conceptualizada como "cualquier comportamiento que implique la humillación, ridiculización, descalificación y burla en público. El agresor se muestra descortés con las amistades y/o familiares de la mujer, e incluso puede seducir a otras mujeres en su presencia" (Gobierno de Cantabria, 2007, p. 14).

Por otra parte, la violencia laboral está referida al ámbito del trabajo donde puede presentarse una obstaculización a su acceso, a una contratación o a una estabilidad o permanencia de este, en el cual se exigen requisitos como la maternidad, el estado civil, edad, apariencia física, realización de pruebas de embarazo o el hostigamiento en el ámbito laboral para su renuncia (Carmona y Veliz, 2005).

La categoría de violencia institucional es otra clasificación, $y$ es ejercida por funcionarios, profesionales, agentes pertenecientes a cualquier órgano, en el que se ha visto y confirmado que desearon retardar, obstaculizar o impedir el acceso a las políticas públicas, ejercer los derechos previstos en la Ley o recibir atención como víctima de algún tipo de violencia de los anteriormente descritos, cuando no se le ha prestado suficiente atención a su denuncia y se ha visto un enlentecimiento o gburla de esta (Ministerio de Justicia y Derechos Humanos, 2011).

Por otro lado, la violencia patrimonial o vicaria (nombre más usado en España) constituye todas aquellas acciones que implican un daño hacia objetos de la persona que recibe violencia, actos como el romper, golpear objetos o eliminar pertenencias de la mujer, buscando incluso destrozar sus enseres, como romper las llantas de su vehículo, dañar su ropa, zapatos u objetos (Huertas, 2012). Este tipo de violencia es de los más dañinos, puesto que el agresor también amenaza con herir o matar a los hijos para generar un daño emocional y psicológico a la pareja o expareja. Esto incluye los malos tratos a infantes por parte de sus progenitores; además, el daño también se puede extender a los animales domésticos de la mujer (Cordero et al., 2017).

Por último, una casi reciente forma de violencia es la de redes sociales, que incluye todo tipo de violencia relacionada con el uso de páginas y perfiles en redes sociales en medios 2.0, donde se pueden publicar aspectos relacionados con la intimidad de la persona (fotos, vídeos) o comentarios que buscan generar en la víctima humillación, burlas, sentimientos de inferioridad, y que van abusivamente a agredir a la persona en 
su autoestima, capacidades intelectuales, aspectos socioeconómicos y personalidad (Arab y Díaz, 2015).

\section{Metodología}

El diseño es cuantitativo, no experimental, con un tipo de estudio descriptivo de corte transversal; se sistematizaron los datos de tipo descriptivo y estadísticas en porcentaje con el software estadístico SPSS (versión 24).

\section{Participantes}

La población de estudio es mujeres víctimas de violencia de género que habían acudido a la Fundación Mujer y Futuro para buscar ayuda legal y psicológica debido a problemas de violencia de género, con edades entre 19 y 69 años, una media de 42,3 años, y una desviación estándar de 16 años.

En este sentido, la muestra fue seleccionada del banco de datos de la Fundación, de acuerdo con unos criterios de inclusión: ser mujeres mayores de edad (18 años), haber tenido episodios de maltrato (1 o más episodios), ser alfabetas, sin diagnóstico psiquiátrico en el momento de la aplicación de la encuesta psicosocial, y no haber sufrido un trauma cerebral o daño cognitivo.

Se convoca a las mujeres que presentaban estas características mediante muestreo no probabilístico, intencional o por conveniencia. De 60 mujeres convocadas, que fue la población con dichas características, 20 mujeres acudieron a las valoraciones. En cuanto a su condición legal, la muestra se caracterizó por estar compuesta por mujeres que habían interpuesto una denuncia por violencia intrafamiliar. 16 de ellas se habían divorciado de su agresor hacía más de 5 años, y 4 de ellas se encontraban con medida de distanciamiento del agresor y estaban bajo el régimen de casa refugio.

\section{Materiales e instrumentos}

Para recopilar los datos se diseñó un instrumento llamado "Encuesta Psicosocial", que se basa en las bases teóricas del International Violence Against Women Survey (IVAWS), que permite esclarecer los tipos de violencia recibidos, características sociodemográficas de la población y aspectos de relaciones familiares de las mujeres víctimas de violencia, con un nivel de confianza del 95 \% (Sagot, 2003; United Nations, 2014).

\section{El IVAWS tiene como objetivo}

[...] proporcionar información sobre la naturaleza y la extensión de la violencia física y sexual infligida contra las mujeres por los hombres en diferentes países del mundo. Esta encuesta forma parte de un proyecto multipaís y transcultural cuyo objetivo primario es promover e implementar la investigación sobre la violencia contra las mujeres en diferentes países del mundo, en particular en países en desarrollo y en transición, como una importante herramienta de investigación y de intervención. (Unidad de Apoyo a la Investigación, 2005, p. 59)

Los países que han participado en la investigación mediante procesos de validación y estandarización de este macroproyecto son "Australia, Canadá, Dinamarca, Inglaterra y Gales, Finlandia, Países Bajos, Suecia, Suiza, Indonesia, Filipinas, Estonia, Kazajstán, Polonia, Ucrania, Argentina, Trinidad y Tobago, Uruguay" (Unidad de Apoyo a la Investigación, 2005, p. 59). También Costa Rica ha participado del proyecto de encuesta IVAWS (Sagot, 2003).

Dentro de las categorías de violencia estudiadas en la encuesta psicosocial diseñada, se encuentran la violencia física, psicológica, sexual, económica, institucional, laboral, patrimonial y/o vicaria, y por redes sociales, conceptos extraídos de las categorías del cuestionario IVAWS y de protocolos sanitarios en materia de violencia de género. La encuesta IVAWS no fue aplicada, dado que no fue posible acceder al contenido completo de esta; por tanto, se desarrolló la "encuesta psicosocial", basada en las bases teóricas de IVAWS y apoyado en artículos que conceptualizan la violencia a través de esta encuesta.

En cuanto a la categoría violencia sexual, se contemplan las subcategorías acoso sexual, explotación sexual, acceso carnal violento, embarazo forzado, negación de la anticoncepción, matrimonio forzado e incesto. Estas violencias fueron tomadas y analizadas por separado a nivel estadístico.

Para la aplicación del instrumento se realizó previamente la firma del consentimiento informado según la Declaración de Helsinki de la Asamblea Médica Mundial (1994), que protege los datos personales y expone el tratamiento de estos, además de dar libertad para poderse retirar en cualquier momento de la investigación.

\section{Procedimiento}

Con la intención de verificar la comprensión de las preguntas en la discriminación de los distintos tipos de violencia, se realizó la validación del instrumento en una sesión en la que participaron 5 estudiantes de psicología, quienes reportaron aspectos asociados a la claridad de este. Posteriormente se inició la fase de convocatoria en la Fundación Mujer y Futuro, donde se hizo una verificación de registro de las mujeres atendidas por violencia de género, y se citó a 60 mujeres mediante llamadas telefónicas, de las cuales asistieron 20.

Para iniciar el proceso de recolección de datos 
se acompañó a las participantes en la lectura del consentimiento informado, se clarificó la libertad y autonomía de participar en la investigación y se reiteró la posibilidad de retirarse en cualquier momento; adicionalmente, se brindó un apoyo económico para gastos relacionados con el traslado. Posteriormente, se procedió a la valoración de las violencias mediante el instrumento encuesta psicosocial. Su aplicación fue realizada en forma de entrevista, en la cual las mujeres podían exponer con detalles los tipos de violencias recibidos, las experiencias vividas y su situación actual. Seguidamente, y de acuerdo con el relato de las participantes durante la entrevista, se clasificaban los tipos de violencia recibidos, y se brindaba un acompañamiento psicológico mediante una contención verbal, de acuerdo con los niveles de malestar emocional referidos.

Una vez finalizada la recolección de la información con la encuesta psicosocial, los datos fueron analizados en el software estadístico SPSS versión 24, que permitió generar las estadísticas descriptivas (frecuencias y porcentajes) relacionadas con los aspectos sociodemográficos y tipos de violencia vividos.

\section{Resultados}

\section{Caracterización sociodemográfica de las mujeres víctimas de la violencia}

La muestra estuvo conformada por 20 mujeres víctimas de violencia de género; población mayormente de adultez temprana $(45,0 \%)$ y adultez intermedia $(45,0 \%)$ (ver Tabla 1).

Tabla 1

Distribución de las mujeres por edad

\begin{tabular}{ccc}
\hline Edad & Frecuencia & Porcentaje \\
\hline $11-20$ & 1 & 5,0 \\
$21-40$ & 9 & 45,0 \\
$41-65$ & 9 & 45,0 \\
$>65$ & 1 & 5,0 \\
Total & 20 & 100,0 \\
\hline
\end{tabular}

Fuente: Autoras.

En cuanto al nivel educativo, la mayor proporción de mujeres víctimas de la violencia encuestadas cuentan con bachillerato (35,0\%); seguidas, con menor proporción, por las que finalizaron la básica primaria $(15,0 \%)$ o tenían primaria incompleta (15,0\%); las mujeres restantes presentan un nivel de formación técnica (10,0\%), universitario incompleto $(10,0 \%)$, formación tecnológica $(5,0 \%)$ y universitario (5,0 \%). En general, más de dos tercios de la población de mujeres no superaba como nivel educativo el bachillerato (70,0 \%) (ver Tabla 2).
Tabla 2

Distribución de las mujeres por nivel educativo

\begin{tabular}{ccc}
\hline Nivel educativo & Frecuencia & Porcentaje \\
\hline Primaria & 3 & 15,0 \\
Primaria incompleta & 3 & 15,0 \\
Bachillerato & 7 & 35,0 \\
Bachillerato incompleto & 1 & 5,0 \\
Técnico & 2 & 10,0 \\
Tecnológico & 1 & 5,0 \\
Universitario & 1 & 5,0 \\
Universitario incompleto & 2 & 10,0 \\
Total & 20 & 100,0 \\
\hline
\end{tabular}

Fuente: Autoras.

Respecto a la estratificación socioeconómica de las mujeres encuestadas, el $100 \%$ se ubica en los primeros 3 estratos, caracterizados por ser los más bajos, en los cuales se ubican las personas con menos recursos económicos en el país. De acuerdo con los resultados de la encuesta presentados en la Tabla 3, las mujeres víctimas de violencia de género por estrato se distribuyen así: el $45 \%$ se ubica en el nivel socioeconómico bajo-bajo, es decir, estrato 1; otro $45 \%$, en un nivel bajo, conocido también como estrato socioeconómico 2 , y una pequeña porción de las encuestadas pertenecen al estrato medio bajo (10\%) o estrato 3 .

Tabla 3

Distribución de las mujeres por estrato socioeconómico

\begin{tabular}{ccc}
\hline Estrato & Frecuencia & Porcentaje \\
\hline Bajo-bajo & 9 & 45,0 \\
(estrato 1) & & \\
Bajo (estrato 2) & 9 & 45,0 \\
Medio-bajo & 2 & 10,0 \\
(estrato 3) & & \\
Total & 20 & 100,0 \\
\hline
\end{tabular}

Fuente: Autoras.

La estratificación socioeconómica en Colombia es un sistema que permite clasificar los bienes inmuebles o viviendas que recibirán los servicios públicos. Básicamente permite diferenciar el cobro de dichos servicios públicos por sectores, en los cuales los de mayor capacidad económica subsidian a los de menor capacidad económica (Departamento Administrativo Nacional de Estadística [DANE], 2020).

La anterior concepción sobre estratificación económica está organizada de acuerdo con la capacidad económica que tienen las personas. En el nivel bajo-bajo, que corresponde al estrato 1 , son personas que perciben menos de un salario mínimo en Colombia, que estaría situado sobre los 200 USD (Fajardo-Calderón et al., 2011). El estrato bajo, que es el estrato 2, está indicado por 
personas que perciben un salario mínimo de 300 USD, y el estrato 3, por personas que perciben entre 300-500 USD mensuales. De acuerdo con los postulados anteriores, los estratos 1,2 y 3 son los que menos perciben ingresos en Colombia.

Por otra parte, respecto al estado civil de las encuestadas, el $45 \%$ de las mujeres víctimas de violencia son separadas; seguidas, con menor proporción, de las mujeres casadas $(35,0 \%)$, en unión libre $(10,0 \%)$, viudas $(5,0 \%)$ y solteras (5,0\%), como se observa en la Tabla 4.

Tabla 4

Distribución de las mujeres por su estado civil

\begin{tabular}{ccc}
\hline Estado civil & Frecuencia & Porcentaje \\
\hline Separada & 9 & 45,0 \\
Casada & 7 & 35,0 \\
Unión libre & 2 & 10,0 \\
Soltera & 1 & 5,0 \\
Viuda & 1 & 5,0 \\
Total & 20 & 100,0 \\
\hline
\end{tabular}

Fuente: Autoras.

Casi dos tercios de la población de mujeres tenía como ocupación ser ama de casa (65,0\%); el tercio restante lo completaban, con similar proporción $(5,0 \%)$, en el momento de la entrevista una estudiante, una vigilante, una guarnecedora de calzado, una auxiliar de enfermería, una trabajadora independiente, una estilista y una auxiliar contable (ver Tabla 5).

Tabla 5

Distribución de las mujeres por su ocupación

\begin{tabular}{ccc}
\hline Ocupación & Frecuencia & Porcentaje \\
\hline Ama de casa & 13 & 65,0 \\
Estudiante & 1 & 5,0 \\
Vigilante & 1 & 5,0 \\
Guarnecedora de calzado & 1 & 5,0 \\
Auxiliar de enfermería & 1 & 5,0 \\
Trabajadora independiente & 1 & 5,0 \\
Estilista & 1 & 5,0 \\
Auxiliar contable & 1 & 5,0 \\
Total & 20 & 100,0 \\
\hline
\end{tabular}

Fuente: Autoras.

En cuanto a las edades en las que inició la violencia en las mujeres de la muestra, el 40 \% comenzó a ser violentada entre los 12 y los 20 años. Esto es, en su etapa de adolescencia y juventud; en tanto un $20 \%$ destacó ser violentada en su primera infancia, y otro $20,0 \%$, en su segunda infancia.

En la Tabla 6 se identifican los distintos tipos de violencia a los que fueron sometidas las mujeres. Dentro de estas se encontró que el $100 \%$ de las mujeres que participaron en la encuesta fueron víctimas de violencia o maltrato psicológico, y es este el porcentaje más alto. De estas, el 80 \% sufrió, además, violencia física; un 70 $\%$, violencia económica, y un $45 \%$, acoso sexual. Estos fueron los resultados más significativos en cuanto al tipo de violencia ocurrido en este grupo de mujeres. Mientras los niveles más bajos de violencia se concentraron en violencia por redes sociales $(5 \%)$, violencia institucional (5\%), incesto (5\%), explotación sexual (5\%), pero se pueden observar importantes valores para la violencia social (40\%), violencia patrimonial (30\%), y acceso carnal violento $(25 \%)$.

\section{Tabla 6}

Tipos de violencia que sufren las mujeres

\begin{tabular}{ccc}
\hline Tipo de violencia & $\begin{array}{c}\text { Frecuencia } \\
\text { de casos }\end{array}$ & $\begin{array}{c}\text { Porcentaje de } \\
\text { respuestas }\end{array}$ \\
\hline Violencia psicológica & 20 & 100,0 \\
Violencia física & 16 & 80,0 \\
Acoso sexual & 9 & 45,0 \\
Explotación sexual & 1 & 5,0 \\
Acceso carnal violento & 5 & 25,0 \\
Embarazo forzado & 0 & 0 \\
Negación anticoncepción & 2 & 10,0 \\
Incesto & 1 & 5,0 \\
Violencia económica & 14 & 70,0 \\
Violencia social & 8 & 40,0 \\
Violencia institucional & 1 & 5,0 \\
Violencia patrimonial & 6 & 30,0 \\
Violencia laboral & 2 & 10,0 \\
Violencia vicaria & 4 & 20,0 \\
Violencia por redes sociales & 1 & 5,0 \\
\hline
\end{tabular}

Fuente: Base de datos grupo de investigadores.

En la Tabla 7 se pueden encontrar las personas que infringieron violencia en estas mujeres. En ella se identifica, entre otros, el ítem denominado "sin definir", que hace alusión a la categoría en la cual las mujeres no desearon identificar al agresor, guardando el secreto por motivos personales, o no sabían identificar el agresor, pues no era conocido, como en el caso de violencia sexual en que la víctima no pudo reconocer a su agresor, pues era de noche y no había luz en la zona donde se encontraba la mujer agredida.

Tabla 7

Generadores de violencia en las mujeres

\begin{tabular}{cccc}
\hline Violencia & Generador & Frecuencia & Porcentaje \\
\hline Acceso carnal & Padrastro & 1 & 20,0 \\
violento & Sin definir & 4 & 80,0 \\
& Total & 5 & 100,0 \\
& Padrastro & 2 & 22,2 \\
& Vecino & 2 & 22,2 \\
Acoso sexual & Suegro & 2 & 22,2 \\
& Cuñado & 2 & 22,2 \\
& Sin definir & 4 & 44,4 \\
& Total & 9 & 100,0
\end{tabular}




\begin{tabular}{|c|c|c|c|}
\hline & Excónyuge & 5 & 35,7 \\
\hline \multirow{3}{*}{$\begin{array}{l}\text { Violencia } \\
\text { económica }\end{array}$} & Cónyuge & 3 & 21,4 \\
\hline & Sin definir & 6 & 42,9 \\
\hline & Total & 14 & 100,0 \\
\hline \multirow{4}{*}{$\begin{array}{l}\text { Explotación } \\
\text { sexual }\end{array}$} & Madre & 1 & 100,0 \\
\hline & Excónyuge & 5 & 31,3 \\
\hline & Madre & 2 & 12,5 \\
\hline & Padrastro & 1 & 6,3 \\
\hline \multirow[t]{4}{*}{ Violencia física } & Padres & 2 & 12,5 \\
\hline & Cónyuge & 3 & 18,8 \\
\hline & Sin definir & 6 & 37,5 \\
\hline & Total & 16 & 100,0 \\
\hline Incesto & Tío & 1 & 100,0 \\
\hline \multirow{2}{*}{$\begin{array}{c}\text { Violencia } \\
\text { institucional }\end{array}$} & Sin definir & 1 & 100,0 \\
\hline & Comunidad & 1 & 50,0 \\
\hline \multirow{4}{*}{ Violencia laboral } & Suegro & 1 & 50,0 \\
\hline & Cuñada & 1 & 50,0 \\
\hline & Total & 2 & 100,0 \\
\hline & Excónyuge & 4 & 66,7 \\
\hline \multirow{6}{*}{$\begin{array}{l}\text { Violencia } \\
\text { patrimonial }\end{array}$} & Cónyuge & 1 & 16,7 \\
\hline & Sin definir & 1 & 16,7 \\
\hline & Total & 6 & 100,0 \\
\hline & Excónyuge & 5 & 25,0 \\
\hline & Madre & 2 & 10,0 \\
\hline & Padrastro & 3 & 15,0 \\
\hline \multirow{5}{*}{$\begin{array}{l}\text { Violencia } \\
\text { psicológica }\end{array}$} & Padres & 4 & 20,0 \\
\hline & Hijo & 1 & 5,0 \\
\hline & Cónyuge & 3 & 15,0 \\
\hline & Sin definir & 7 & 35,0 \\
\hline & Total & 20 & 100,0 \\
\hline \multirow{2}{*}{$\begin{array}{l}\text { Violencia en } \\
\text { redes sociales }\end{array}$} & Sin definir & 1 & 100,0 \\
\hline & Excónyuge & 4 & 50,0 \\
\hline \multirow{4}{*}{ Violencia social } & Cónyuge & 1 & 12,5 \\
\hline & Sin definir & 3 & 37,5 \\
\hline & Total & 8 & 100,0 \\
\hline & Excónyuge & 1 & 25,0 \\
\hline \multirow[t]{3}{*}{ Violencia vicaria } & Padre & 1 & 25,0 \\
\hline & Sin definir & 2 & 50,0 \\
\hline & Total & 4 & 100,0 \\
\hline
\end{tabular}

Fuente: Autoras.

Por su parte, en la Tabla 8 se observa que cuatro quintas partes de las mujeres encuestadas se vieron sometidas a violencia en el núcleo familiar (80,0\%).

Tabla 8

Violencia en núcleo familiar

\begin{tabular}{ccc}
\hline & Frecuencia & Porcentaje \\
\hline No & 4 & 20,0 \\
Sí & 16 & 80,0 \\
Total & 20 & 100,0 \\
\hline
\end{tabular}

Fuente: Autoras.

La encuesta psicosocial aplicada presentó un alfa de Cronbach de 0,636, que es un puntaje moderadoaceptable (Bonilla-Escobar et al., 2013; Cortina, 1993), comprendiendo que es un primer pilotaje y aproximación al estudio de la violencia de género.

\section{Discusión}

Según la caracterización expuesta en este estudio, se encontraron factores como baja escolaridad, dependencia económica por parte de la pareja y malos tratos desde el hogar base (disfuncionalidad familiar), como aspectos que generan vulnerabilidad a la mujer para vivir situaciones de violencia. Estos factores también fueron encontrados en otros estudios, como los de Ambriz-Mora et al. (2015); Díaz et al. (2015); y Jaen et al. (2015). Además, el alto consumo de alcohol por parte del cónyuge puede predisponer a la violencia en el hogar (Díaz et al., 2015), factor que no fue tenido en cuenta en este estudio.

Adicionalmente, en el estudio de Cruz et al. (2013), se asociaron factores como el nivel socioeconómico bajo y la escolaridad básica con la violencia hacia la mujer, factores que también se visibilizaron en el presente estudio, en el que las mujeres encuestadas presentaron niveles socioeconómicos muy bajos y poca preparación, con los porcentajes más altos. Esto implica que para muchas mujeres la falta de independencia económica y el bajo nivel académico pueden influir en su empoderamiento laboral y social (Casique, 2010; Hidalgo y Valdés, 2014), por lo que se sugiere la presencia de una relación de dependencia económica y afectiva que vulnera las relaciones de la mujer con su cónyuge, la familia y la sociedad (Aiquipa, 2015; Casique, 2010).

También es importante reconocer que las mujeres evaluadas en su mayoría se dedican a las labores del hogar como amas de casa, sin ingresos como independientes; resultados muy coherentes con los de otras investigaciones que indican que las mujeres que poseen ingresos económicos bajos sufren mayor violencia (Calderón et al., 2011; González y Fernández, 2010; Puente-Martínez et al., 2016). Otras investigaciones manifiestan que un factor protector está vinculado con tener un nivel académico alto, ya que protege a las mujeres de sufrir violencia intrafamiliar, por las capacidades de negociación y manejo de situaciones que pueden adquirirse con mayores estudios, además de las mejoras en la economía familiar (Calderón et al., 2011; Castañeda et al., 2009; Castro y Casique, 2009; González y Fernández, 2010; Machado et al., 2009; Ramírez y Núñez, 2010).

Adicionalmente, se ha demostrado que cuando la violencia se inicia en la infancia suele prolongarse hasta la adolescencia e incluso hasta la adultez (Flórez y González, 2013). En este estudio la mayoría de las mujeres víctima de violencia de género provenían de hogares donde recibieron malos tratos desde la infancia ( $80 \%$ ), y también formalizaron relaciones sentimentales a muy temprana edad. Según las narraciones desarrolladas, salían de casa por problemas de violencia y bajo apoyo social, situación 
que también puede comprobarse en el informe de Garmendia (2011), donde los actos violentos tienen un origen en relaciones familiares núcleo disfuncionales, o haber sufrido malos tratos desde la infancia por parte de sus progenitores. Estos actos violentos y relaciones de pareja a temprana edad por parte de las mujeres pudieron haber facilitado la legitimación del ejercicio de dominación mediante el acto violento, evaluando los malos tratos como algo posible y aceptable en la relación, que privilegia la estabilidad familiar (ÁlvarezDardet et al., 2013; Preciado-Gavidia et al., 2010).

En otros estudios también se confirma que recibir malos tratos desde la infancia normaliza la violencia en las relaciones y la disfuncionalidad en familias violentas (Espinosa et al., 2011; Gonzales et al., 2013).

Por otro lado, en esta investigación la violencia psicológica fue la más frecuente, presentándose en las 20 mujeres encuestadas (100\%). Resultados similares fueron encontrados por Akl-Moanack et al. (2016) y Espinosa et al. (2011), quienes hallaron un porcentaje significativo de la población femenina víctima de este tipo de violencia (60\% y $100 \%$, respectivamente). Los autores, además, resaltaron que todo acto de violencia física estaba acompañado de violencia emocional o psicológica; resultados apoyados por otras investigaciones, como la de Cruz et al. (2013), quienes encontraron que la violencia psicológica fue la más frecuente, seguida de la violencia sexual, lo que sugiere que las víctimas suelen recibir con frecuencia violencia psicológica que vulnera su autoestima y lesiona sus capacidades emocionales de supervivencia, y esto facilita otros tipos de violencias.

Por su parte, la violencia física se muestra alta para este estudio, igual que lo reportado por Akl-Moanack et al. (2016) en un $60 \%$, seguida de violencia económica y sexual. Generalmente este tipo de violencias es descrita en varios estudios como la que se suele infringir contra las mujeres (Akl-Moanack et al., 2016; Castro y Casique, 2009; Espinosa et al., 2011; Machado et al., 2009). Esto indica que las mujeres reciben violencia a tan gran escala, de modo que se hace necesario el desarrollo de programas que ayuden a mitigar los daños en la salud mental que producen los actos violentos en el mundo, considerándolo como un problema de salud pública que requiere urgente atención, debido a su gravedad (Aiquipa, 2015; Calderón et al., 2011; Casique, 2010; González y Fernández, 2010; Medrano et al., 2017; Puente-Martínez et al., 2016).

En Colombia se ve la imperiosa necesidad de desarrollar programas que puedan apoyar el impacto que estas violencias generan en las mujeres, así como de reeducar a la población para la prevención de violencia como única forma de resolver conflictos y dañar psicológica, física y económicamente a las mujeres (Ruta pacífica de las mujeres, 2013; Walker, 2012; Wilches, 2010).

\section{Conclusiones}

Los resultados muestran cómo todas las mujeres fueron violentadas psicológicamente, y esta violencia es una de las más nocivas para la salud mental; además, vulnera a la mujer hacia otras formas de violencia.

El abuso sexual también mostró un alto nivel de frecuencia en las mujeres de este estudio, lo que indica que existen factores que predisponen o vulneran derechos protectores en estas mujeres que pudiesen evitar el sufrir este tipo de violencias.

La violencia económica se muestra con alto porcentaje, lo que indica que las parejas sentimentales de este grupo de mujeres no cumplían con la responsabilidad económica y, por ende, se sugiere que esta situación produjo una carencia de necesidades básicas que pueden predisponer a una vulnerabilidad no solo de la mujer, sino de los hijos.

Por su parte, la violencia física aún marca una pauta alta en la estadística: indica que no existen formas asertivas de lidiar con situaciones problemáticas en la casa, además del uso de la violencia para perpetuar relaciones de poder inequitativas.

Como posibles factores vulneradores para que una mujer sea receptora de violencias intrafamiliares, de acuerdo con otros estudios, están la baja escolarización, percibir bajos o nulos apoyos económicos, haber recibido violencia desde el hogar núcleo por padre, madre, tíos u otros, y tener un nivel socioeconómico bajo.

Como factor protector, y de acuerdo con otros estudios, se destaca la educación a niveles superiores, que se muestra como una variable que puede apoyar el manejo y resolución de conflictos de forma satisfactoria $y$, por ende, también obtener mejores recursos económicos.

La violencia es un fenómeno social tan dañino que afecta la salud pública y no permite romper el círculo social del maltrato; por ende, las políticas de salud pública deberán hacer mayor énfasis en mejorar esta problemática social con planes de acción eficaces.

\section{Agradecimientos}

Se agradece a la Universidad Cooperativa de Colombia por su aporte económico en el proyecto de investigación "Violencia hacia las mujeres por razones de género y postconflicto", con centro de costos INV 1788. A la Fundación Mujer y Futuro, por su apoyo en la consecución de la población. A las estudiantes de pregrado Yaneth 
Licte Avalo Ortega, Kely Susana Jaimes Rincón y Leidy Tatiana Ortega, por sus aportes en la investigación.

\section{Referencias}

Aiquipa, J. (2015). Dependencia emocional en mujeres víctimas de violencia de pareja. Revista de Psicología (PUCP), 33(2), 411-437. http://www.scielo.org. pe/scielo.php?script=sci_arttext\&pid=S025492472015000200007\&lng=es\&tlng=es

Akl-Moanack, P., Jiménez, E. y Aponte, F. (2016). Estrategias de afrontamiento en mujeres víctimas de violencia intrafamiliar. Cultura Educación y Sociedad, 7(2), 105-121. https://revistascientificas.cuc.edu. co/index.php/culturaeducacionysociedad/article/ view/1105

Álvarez-Dardet, S., Pérez, J. y Lorence, B. (2013). La violencia de pareja contra la mujer en España: Cuantificación y caracterización del problema, las víctimas, los agresores y el contexto social y profesional. Psychosocial Intervention, 22(1), 41-53. https://doi.org/10.5093/in2013a6

Ambriz-Mora, M. I., Zonana-Nacach, A. y AnzaldoCampos, M. C. (2015). Factores asociados a violencia doméstica en mujeres mexicanas vistas en primer nivel atención. Semergen, 41(5), 241-246. https://doi. org/10.1016/j.semerg.2014.07.004

Andrade, J., Parra, M. y Torres, D. (2013). Desplazamiento forzado y vulnerabilidad en la salud mental en Colombia. Revisión del estado del arte. Poiésis, 25, $1-16$.

Añón, M. J. (2016). Violencia con género. A propósito del concepto y la concepción de la violencia contra las mujeres. Cuadernos Electrónicos de Filosofía Del Derecho, (33), 1-26. https://doi.org/10.7203/ CEFD.33.8257

Arab, E. y Díaz, A. (2015). Impacto de las redes sociales e internet en la adolescencia: Aspectos positivos y negativos. Revista Médica Clínica Las Condes, 26(1), 7-13.

Asamblea Médica Mundial. (1994). Declaración de Helsinki de la Asociación Médica Mundial. Recomendaciones para guiar a los médicos en la investigación biomédica en personas. https://www.uchile.cl/portal/investigacion/ centro-interdisciplinario-de-estudios-en-bioetica/ documentos/76030/declaracion-de-helsinki-de-laasociacion-medica-mundial

Bell, V., Méndez, F., Martínez, C., Palma, P. P. y Bosch, M. (2012). Characteristics of the Colombian Armed Conflict and the Mental Health of Civilians Living in Active Conflict Zones. Journal Conflict and Health, 6(1), 10. https://doi.org/10.1186/1752-1505-6-10

Bonilla-Escobar, F. J., Bonilla-Vélez, J., Ángel-Isaza, A. M. y Ortega-Lenis, D. (2013). Desarrollo y validación de un cuestionario para la caracterízación de estudiantes de medicina investigadores en Colombia. Archivos de Medicina, 9(1:2), 1-7.
Calderón, V., Gáfaro, M.y lbáñez, A. (2011). Desplazamiento forzoso, participación laboral femenina y poder de negociación en el hogar: ¿Empodera el conflicto a las mujeres? (N.o 009252).

Carmona, K. y Veliz, H. (2005). Discriminación Laboral [tesis de grado, Universidad de Chile]. Repositorio Universidad de Chile, http://repositorio.uchile.cl/ tesis/uchile/2005/carmona_k/sources/carmona_k. pdf

Casique, I. (2010). Factores de empoderamiento y protección de las mujeres contra la violencia. Revista Mexicana de Sociología, 72(1), 37-71. http://www.scielo. org.mx/scielo.php?script=sci_arttext\&pid=S018825032010000100002\&lng=es\&tlng=es

Castañeda, M. ., Tamayo, M. ., Miraval, Z. ., Manrique, D., Alvarado, N. y Carillo, C. (2009). Perspectiva de las mujeres acerca de la violencia basada en género, como factor de empobrecimiento. Revista Peruana de Ginecología Obstetricia, 55(4), 256-259.

Castro, R.y Casique, I. (2009). Violencia de pareja contra las mujeres en México: una comparación entre encuestas recientes. México: Notas de Población, núm. 87.

Cea, P. (2015). De víctimas a agentes: imaginarios y prácticas sobre la violencia de género en la pareja contra mujeres inmigrantes en el Estado español. [tesis de doctorado, Universitat Autónoma de Barcelona]. https://dialnet. unirioja.es/servlet/tesis?codigo $=117367$

Chejter, S. y Isla, V. (2018). Abusos sexuales y embarazo forzado en la niñez y adolescencia: lineamientos para su abordaje interinstitucional (Primera ed). https://www. unicef.org/argentina/informes/abusos-sexuales-yembarazo-forzado-en-la-niñez-y-adolescencia

Contreras, L. (2014). Factores de Riesgo de Homicidio a la Mujer en la Relación de Pareja. Universitas Psychologica, 13(2), 681-692. https://revistas.javeriana.edu.co/ index.php/revPsycho/article/view/3192

Cordero, G., López, C. y Guerrero, A. (2017). Otra forma de violencia de género: la instrumentalización. "Donde más te duele." Documentos de Trabajo Social: Revista de Trabajo y Acción Social, (59), 1133-6552.

Cortina, J. M. (1993). What is Coefficient Alpha? An Examination of Theory and Applications. Journal of Applied Psychology, 78(1), 98-104.

Cruz, A., Morales-Ramón, F., Arriaga-Zamora, R. y ChanGarcía, A. (2013). Prevalencia de violencia conyugal en mujeres de 15 a 45 años en una localidad de Balancán, Tabasco. Salud en Tabasco, 19(2), 44-50.

Departamento Administrativo Nacional de Estadística [DANE]. (2020). Estratificación socioeconómica para servicios públicos domiciliarios. https://www.dane. gov.co/index.php/servicios-al-ciudadano/serviciosinformacion/estratificacion-socioeconomica

Díaz, S., Arrieta, K. y González, F. (2015). Violencia intrafamiliar y factores de riesgo en mujeres afrodescendientes de la ciudad de Cartagena. Revista Clínica de Medicina de Familia, 8(1), 19-30. https://doi.org/10.4321/S1699-695X2015000100004 
Espinosa, M., Alazales, M., Madrazo, B., García, A. y Presno, M. (2011). Comportamiento de la violencia doméstica en mujeres pertenecientes al consultorio "Altos de Milagro." Revista Cubana de Medicina General Integral, 27(2), 261-269.

Fajardo-Calderón, C. L., Jaramillo, R. A. y Gómez, E. (2011). Los ingresos de las familias de los estratos 1 y 2 de la Comuna Diez del municipio de Armenia y su impacto en el consumo de los alimentos de la canasta básica familiar. Criterio Libre, 9(14), 237-314.

Flórez, M. y González, A. (2013). Caracterización de la violencia intrafamiliar, maltrato infantil y abuso sexual en Bogotá D.C. durante el año 2011. Teoría y Praxis Investigativa, 8(1), 74-91.

Garmendia, L. (2011). La violencia en América Latina. Anales de La Facultad de Medicina, 72(4), 269-276.

Gobierno de Cantabria. (2007). Violencia contra las mujeres. Protocolo de actuación ante los malos tratos (Segunda ed.). Consejería de Sanidad, Dirección Nacional de Salud Pública.

Gonzales, J., Rivas, F., Marín, X. y Villamil, L. (2013). Niveles de disfunción familiar en veinte mujeres víctimas de violencia intrafamiliar el municipio de Armenia. El Ágora U.S.B., 13(2), 399-410.

González, G. y Bejarano, R. (2014). La violencia de género: evolución, impacto y claves para su abordaje. Enfermería Global, 13(33), 424-439.

González, H. y Fernández, T. (2010). Género y maltrato: violencia de pareja en los jóvenes de Baja California. Estudios Fronterizos, 11(22), 97-128.

Guaderas Alburja, P. (2015). La intervención psicosocial contra la violencia de género en Quito.Tejiendo narrativas y nuevos sentidos [tesis de doctorado, Universidad Autónoma de Barcelona]. https://www. tdx.cat/bitstream/handle/10803/385738/pga1de1. pdf? sequence $=1$

Hewitt, N., Juárez, F., Parada, A. J., Guerrero, J., Romero, Y. M., Salgado, A. M. y Vargas, M. V. (2016). Afectaciones psicológicas, estrategias de afrontamiento y niveles de resiliencia de adultos expuestos al conflicto armado en Colombia. Revista Colombiana de Psicología, 25(1), 125-140. https://doi.org/10.15446/ rcp.v25n 1.49966

Hidalgo, L. y Valdés, D. (2014). Violencia contra la mujer adulta en las relaciones de pareja. Medisan, 18(2), 181-187.

Huertas, O. (2012). Violencia intrafamiliar contra las mujeres. Revista Logos, Ciencia y Tecnología, 4(1), 96-106.

Jaen, C., Rivera, S., Amorin, E.y Rivera, L. (2015). Violencia de pareja en mujeres: prevalencia y factores asociados. Acta de Investigación Psicológica, 5(3), 2224-2239. https://doi.org/10.1016/s2007-4719(16)30012-6

Lozano, N. (2016). Boletín Epidemiológico. Violencia de género en Colmbia. Análisis comparativo de las cifras de los años 2014, 2015 y 2016. Instituto Nacional de Medicina Legal y Ciencias Forenses https://www. medicinalegal.gov.co/cifras-estadisticas/boletinesepidemiologicas

Machado, C., Caridade, S. y Martins, C. (2009). Violence in Juvenile Dating Relationship Self-reported Prevalence and Attitudes in a Portugal Simple. Journal of Family Violence, 25(1), 43-52.

Medrano, A., Miranda, M. y Figueras, V. (2017). Violencia de pareja contra las mujeres en México: una mirada a la atención del sector salud desde una perspectiva interseccional. Multidisciplinary Journal of Gender Studies, 6(1), 1231-1262. https://doi.org/10.17583/ generos.2017.2131

Ministerio de Justicia y Derechos Humanos. (2011). Comisión Nacional Coordinadora de Acciones para la Elaboración de Sanciones de Violencia de Género (CONSAVIG). https://www.argentina.gob.ar/ consavig

Navarro, J. (2015). Violencia en las relaciones íntimas, una perspectiva clinica. Herder Editorial.

Observatorio de igualdad de género de America Latina y el Caribe. (2018). Nota para la Igualdad n.o 27: El feminicidio, la expresión más extrema de la violencia contra las mujeres. https://oig.cepal.org/es/notas/ nota-la-igualdad-no-27-feminicidio-la-expresionmas-extrema-la-violencia-mujeres

Orjuela, L. y Rodríguez, V. (2012). Violencia sexual contra los niños y las niñas. Abuso y explotación sexual infantil. SAVE THE CHILDREN ESPAÑA.

Preciado-Gavidia, P. M., Torres-Cendales, N. E. y ReyAnacona, C. A. (2010). Mujeres que finalizaron una relación maltratante: características de personalidad, psicopatológicas y sociodemográficas. Universitas Psychologica, 11(1), 43-54. https://doi.org/10.11144/ Javeriana.upsy11-1.mfrm

Puente-Martínez, A., Ubillos-Landa, S., Echeburúa, E. y Páez-Rovira, D. (2016). Factores de riesgo asociados a la violencia sufrida por la mujer en la pareja: una revisión de meta-análisis y estudios recientes. Anales de Psicología, 32(1), 295-306. https://doi. org/10.6018/analesps.32.1.189161

Ramírez, C. y Núñez, D. (2010). Violencia en la relación de noviazgo en jóvenes universitarios: un estudio exploratorio. Enseñanza e Investigación en Psicología, 15(2), 273-283.

Ribeiro, W., Andreoli, S., Ferri, C., Prince, M. y Mari, J. (2009). Exposure to Violence and Mental Health Problems in Low and Middle-Income Countries: A Literature Review. Rev Bras Psiquiatr, (2), 49-57.

Rodríguez, A. y lbarra, M. E. (2013). Los estudios de género en Colombia. Una discusión preliminar. Sociedad y Economía, (24), 15-46.

Ruta pacífica de las mujeres. (2013). La verdad de las mujeres víctimas del conflicto armado en Colombia (Tomo II). G2 Editores.

Sagot, M. (2003). Encuesta Internacional de violencia contra las mujeres IVAWS. https://issuu.com/pnudsv/ docs/montserrat_sagot_parte_ii._un_recor 
Sánchez, L., Baena, Y., Montoya, W. y Fernández, S. (2013). Aportes de la psicología jurídica en el abordaje de la violencia conyugal hacia la mujer. Revista Psicoespacios, 7(11), 288-306. http://revistas.iue.edu. co/index.php/Psicoespacios

Sanz, M. y Sellarés, J. (2010). Detección de la violencia de género en atención primaria. Revista Española de Medicina Legal, 36(3), 104-109.

Servicio Andaluz de Salud. (2019). Personal sanitario y no sanitario del SAS. Ediciones Rodio.

Unidad de Apoyo a la Investigación. (2005). Catálogo de instrumentos para cribado y frecuencia del maltrato físico, psicológico y sexual. https://www.mscbs.gob. es/organizacion/sns/planCalidadSNS/e02_t03_ Instrumentos.htm

United Nations. (2014). Guidelines on Producing Statistics on Violence Against Women: Statistical Surveys. https://unstats.un.org/unsd/gender/docs/ Guidelines_Statistics_VAW.pdf

Vieyra, C., Gurrola, G. M., Balcázar, P., Bonilla, M. P. y Vírseda, J. A. (2009). Estado de salud mental en mujeres víctimas de violencia conyugal que acuden a la Procuraduría General de Justicia del Estado de México. Psicología lberoamericana, 17(1), 57-64.

Walker, L. E. (2012). El síndrome de la mujer maltratada. Desclee de Brouwer.

Wilches, I. (2010). Lo que hemos aprendido sobre la atención a mujeres víctimas de violencia sexual en el conflicto armado colombiano. Revista de Estudios Sociales, (36), 86-94. 